\title{
OPTIMIZATION OF WOOD MACHINING PARAMETERS IN CNC ROUTERS: TAGUCHI ORTHOGONAL ARRAY BASED SIMULATED ANGLING ALGORITHM
}

\author{
Ender Haziri,^, Kücük Hüseyin Koc ${ }^{2}$
}

\begin{abstract}
In the present study, two mathematical models were developed to optimize the surface roughness for machining condition of Cedar of Lebanon pine (Cedrus libani). Taguchi approach was applied to examine the effect of CNC processing variables. Quality characteristics parameters were selected as arithmetic average roughness $\left(R_{\mathrm{a}}\right)$ and average maximum height of the profile $\left(R_{z}\right)$ for wood material. Analysis of variance (ANOVA) was used to determine effective machining parameters. Developed mathematical models using response surface methodology (RSM) were optimized by a combined approach of the Taguchi's $L_{27}$ orthogonal array based simulated angling algorithm (SA). Optimum machining levels for determining the minimum surface roughness values were carried out three stages. Firstly, the desirability function was used to optimize the mathematical models. Secondly, the results obtained from the desirability function were selected as the initial point for the simulated angling algorithm. Finally, the optimum parameter values were obtained by using simulated angling algorithm. Minimum $R_{a}$ value was obtained spindle speed of $17377 \mathrm{rpm}$, feed rate of 2,012 m/min, tool radius of $8 \mathrm{~mm}$ and depth of cut of 2,009 $\mathrm{mm}$ by using desirability function based simulated angling algorithm. For $R_{z}$ these results were found as $16980 \mathrm{rpm}, 2,004 \mathrm{~m} / \mathrm{min}, 8,001 \mathrm{~mm}$ and $2,003 \mathrm{~mm}$. The $R$-square values of the $R_{a}^{z}$ and $R_{z}$ were $95,91 \%$ and $96,12 \%$, respectively. The proposed models obtained the minimum surface roughness values and provided better results than the observed values.
\end{abstract}

Keywords: Cedrus libani, response surface method, softwood, surface roughness, wood material.

\section{INTRODUCTION}

Wood surface roughness is a crucial indicator of the quality of CNC processing parameters. However, wood material has very complex structure parameters such as machining procedure, physical properties of panel, processing conditions and anatomical structure (Magoss 2008, Philbin and Gordon 2006, Hiziroglu and Kosonkorn 2006, Ozdemir and Hiziroglu 2007, Ratnasingman and Scholz 2006, Hazir and Ozcan 2019). For this reason, it is difficult and complex to investigate the optimum parameter levels. A systematic approach could be applying to provide the optimum process conditions. Therefore, a novel experimental design is required to reduce the number of experiment. Traditional experimental design was required have a long time and it increased the cost. Design of experiment (DOE) and the Taguchi methodology are powerful techniques to reduce the number of experiments. Moreover, these methods have been commonly applied in different engineering applications (Yang and Tarng 1998, Taguchi et al. 2005, Sarikaya and Güllü 2016, Rao and Murthy 2018, Selaimia et al. 2017, Azhiri et al. 2014, Majumder et al. 2017, Kant and Sangwan 2014, Deepanraj et al. 2017). In the recent years, metaheuristic algorithms have been applied to various engineering problems. Especially, experimental design based metaheuristic algorithms have become popular method for optimizing the param-

${ }^{1}$ Faculty of Forestry, Department of Forest Industry Engineering, Istanbul University-Cerrahpasa, Istanbul, Turkey.

${ }^{2}$ Faculty of Forestry, Department of Forest Industry Engineering, Istanbul University-Cerrahpasa, Istanbul, Turkey. hkoc@istanbul.edu.tr "Corresponding author: ender.hazir@istanbul.edu.tr

Received: 11.08.2018 Accepted: 12.06.2019 
eters (Rao and Kalyankar 2013, Mahes et al. 2015, Samanta 2009). Coelho et al. (2008) applied the Taguchi technique to evaluate the effective parameters for wood cutting conditions. Gaitonde et al. (2008) studied the Taguchi design to optimize drilling parameters such as cutting speed and feed rate to reduce delamination of medium density fiberboard (MDF). The delamination was reduced higher cutting speed and lower feed rate. Davim et al. (2009) studied the determination of cutting factors such as cutting speed and feed rate on surface roughness in MDF milling. According to the results, the wood surface roughness decreased with an increase of spindle speed and increased with the feed rate. Prakash and Palanikumar (2010) applied the Taguchi approach to find the effective ratio on $R_{a}$ for drilling MDF. The drilling parameters were spindle speed, feed rate and drill diameter. According to the Taguchi analysis, the feed rate was found to be the most important factor affecting surface roughness. Sofuoglu (2017) investigated that the optimization of CNC machining parameters were examined using the Taguchi method on the surface quality of massive wooden panels made of Scots pine (Pinus slyvestris). Optimal cutting results of $R_{a}$ and $R_{z}$ were resulted with cutter 1 , at a tool clearance strategy of a raster spindle speed of $16000 \mathrm{rpm}$, feed rate of $1000 \mathrm{~mm} / \mathrm{min}$ and depth of cut of $4 \mathrm{~mm}$. Hazir et al. (2018) used $2^{k}$ full factorial design to determine the significant variables and optimum processing levels to determine the lower the wood surface roughness. As a result of this study, two equations were created for radial and tangential cutting directions. Moreover, each models were solved by applying desirability function. Koç et al. (2017) applied full factorial design to find the significant variables on wood surface roughness. Surface roughness values were determined by using laser integrated measurement system and stylus type equipment. According to this study, the roughness values for these measurement methods were found similar. Wilkowski et al. (2011) used the Taguchi technique to determine CNC processing factors for wood surface roughness. The parameters such as feed rate and spindle speed were investigated. Asiltürk et al. (2016) used the Taguchi orthogonal array and RSM to determine the effective factor. Moreover, the effective parameters were optimized for CNC lathe machining. Zhou et al. (2017) applied multi objective optimization technique by using grey relation analysis (GRA), radial basis function (RBF) neural network and particle swarm algorithm. In this study, workpiece material was selected as Ni-based superalloy Inconel 718. According to results, presented method was determined as successfully for multi-axis ball-end milling process. Zhou et al. (2016) used response surface methodology and genetic algorithm to optimize the surface roughness of nickel-based single crystal superalloy using microgrinding process. According to verification results, optimum parameter levels were found as reliable. Bharathi and Baskar (2011) proposed the particle swarm optimization (PSO) algorithm to analyze the optimal processing factors for minimizing the surface roughness and processing time using aluminum material. Parameters of cutting speed, feed, and depth of cut were optimized by using PSO. They indicated that presented method was efficient for machining process. Bao et al. (2018) predicted wood surface roughness conducting the neural network for wood sanding process. They reported that presented model was usefully predicting the roughness value. According to previous studies, the effective parameters on the wood surface roughness are defined in cause and effect diagram given in (Figure 1).

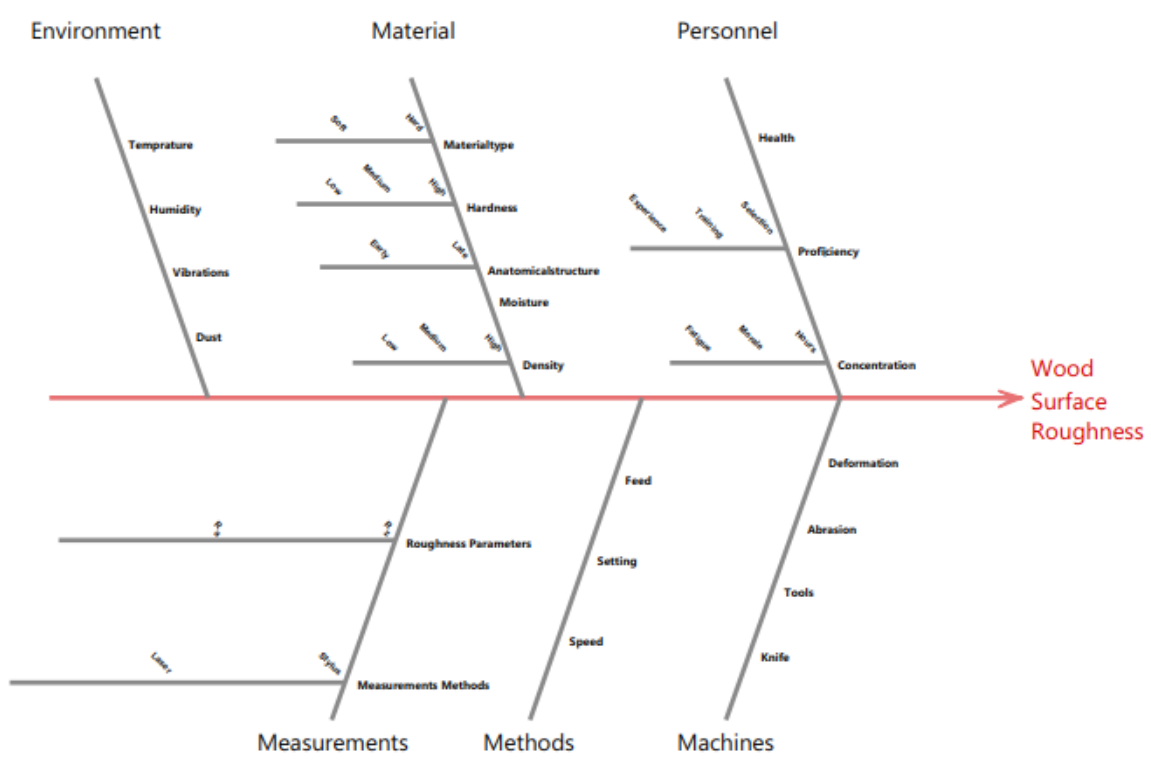

Figure 1: Cause and effect diagram of wood surface roughness parameters. 
$\mathrm{CNC}$ processing parameters and their effects on wood surface roughness were determined in various past studies. However, there is little as no information evaluating the optimum cutting parameters for woodworking industries. Most of these studies presented various engineering problems not inherited to wood based products. Therefore, this study aims to present an approach for determining minimum surface roughness by integrating $\mathrm{RSM}, \mathrm{DF}$ and $\mathrm{SA}$ in CNC processing.

\section{MATERIALS AND METHODS}

Cedar of Lebanon pine (Cedrus libani A. Rich) species with intensive use in the furniture industry was selected for the study. The samples were prepared with the dimension of $140 \mathrm{~mm}$ x $50 \mathrm{~mm}$ x $18 \mathrm{~mm}$ for each procedure. Density level of Cedar of Lebanon pine was measured randomly through 27 samples. Samples were conditioned in a climate room having a temperature of $20^{\circ} \mathrm{C}$ and relative humidity of $65 \%$ until they reach a moisture content of $9 \pm 1 \%$. The value of wood density was $510 \mathrm{~kg} / \mathrm{m}^{3}$. These samples were processed with 3-axis CNC. Alpha-CAM program was used for determination of the tool path for the sample. Roughness measurement device is a stylus-based portable profilometer that is Sutronic-25 type equipment. Parameters of $R_{a}$ and $R_{z}$ were used to evaluate the wood surface quality (ISO 4287-1997).

\section{Statistical design of experiment}

The statistical analysis was performed using Minitab software package 17. The Taguchi $L_{27}$ orthogonal statistical designs were followed to optimize the experimental procedure. The processing variables were screened by ANOVA analysis. These factors were modeled by using response surface methodology (RSM). The results were analyzed by multiple regression analysis through least square method. All the terms of model were tested and verified statistically by $F$-test at probability levels $(p<0,05) . R$-square and $A d j$ - $R$-square were used to perform the developed the models. After the fitting models, 3D surface plots were applied to evaluate the process parameters. Finally, desirability function based simulated angling algorithm was used to screen the optimum parameter levels. The experimental produce is shown in (Figure 2).
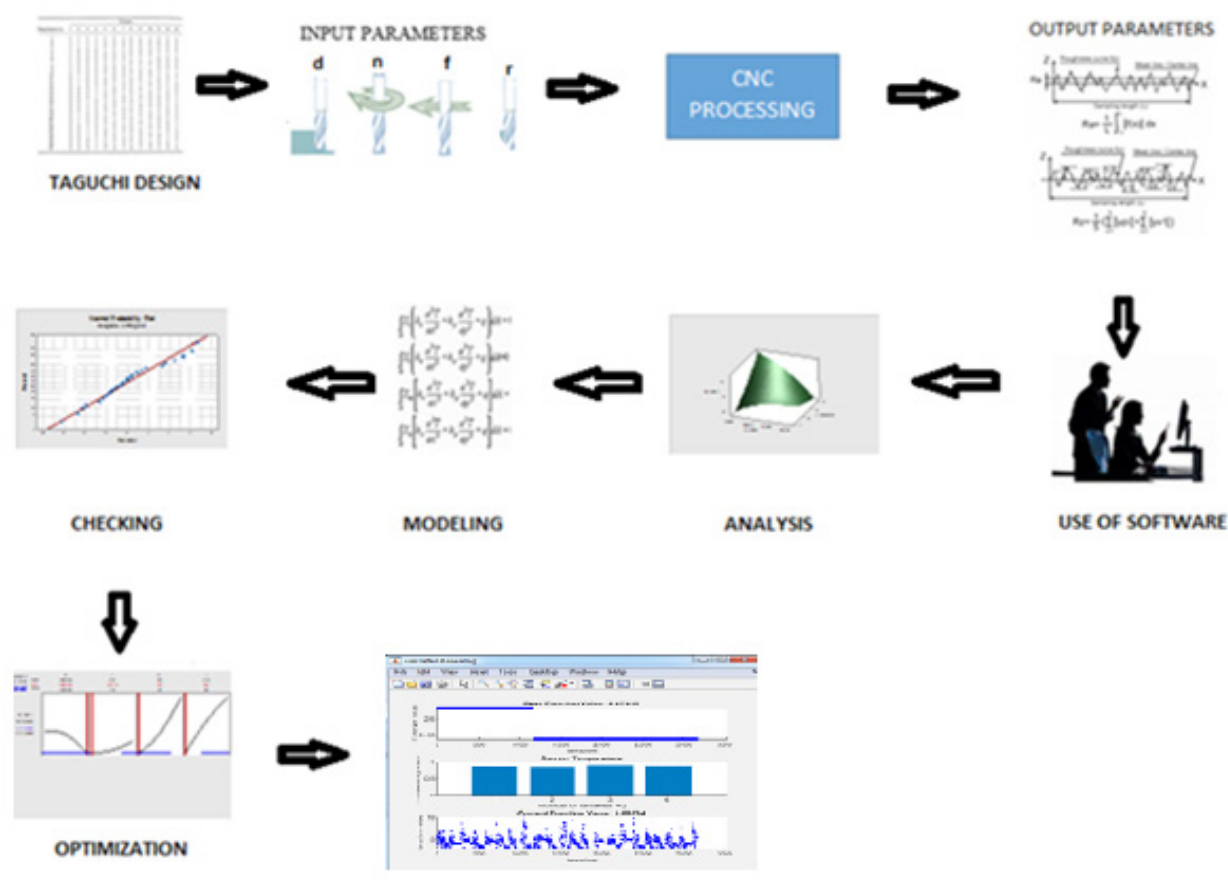

Figure 2: Experimental procedure. 


\section{Response surface method (RSM)}

Response surface method (RSM) is a statistical based technique. RSM method involves three stages. The first stage is the selection of independent variables of major effects on the process through screening studies and the delimitation of the experimental region. The second stage is the mathematic-statistical apply of the obtained experimental data through the fit of a polynomial function and evaluation of the model's fitness. The third stage is the verification of the necessity and possibility of performing a displacement in direction to the optimal region and selection of optimum values of parameters. The general form second order polynomial equation is given Equation 1:

$$
Y=\beta_{0}+\sum_{i=1}^{k} \beta_{i} X_{i}+\sum_{i=1}^{k} \beta_{i i} X_{i}^{2}+\sum_{i} \sum_{j}^{k} \beta_{i j} X_{i} X_{j}+\grave{o}
$$

According to Equation $1 \mathrm{Y}$ is the predicted reaction or reactions $\left(R_{a}\right.$ and $\left.R_{z}\right), X_{i}$ and $\underline{X}_{i}$ are variables, $\beta_{0}$ a constant, $\beta_{\mathrm{i},} \beta_{\mathrm{ii}}$ and $\beta_{\mathrm{ij}}$ of linear, quadratic and the second-order terms, respectively and $k$ is the number of independent parameters $(\mathrm{k}=4$ in this study) and $\epsilon$ is the error term.

\section{Experimental design}

The traditional experimental design is time consuming and costly. $L_{27}$ orthogonal design was used to overcome this issue. Especially, this method is used by manufacturing process and engineering analysis. These arrays of different combination for variables were applied by the Taguchi approach to analyze the experimental study. While 81 experiments are required to investigate all the effects of the parameters, 27 experiments are sufficient thanks to Taguchi orthogonal array. According to the Taguchi orthogonal array, the minimum number of experiment and coded variables were computed by using Equation 2 and Equation 3.

The Taguchi experiments trial number:

$$
[(L-1) x P]+1
$$

The terms of $\mathrm{L}$ and $\mathrm{P}$ are displayed number of levels and number of parameters.

$$
X_{i}=\frac{x_{i}-x_{o}}{\Delta_{x}}
$$

In terms of $X_{i} X_{o}$ and $\Delta_{\mathrm{x}}$ are coded variable, actual variable, center palace, and altered variable. (Table 1) shows the number of parameters and their levels.

Table 1: CNC experimental design procedure.

\begin{tabular}{|c|c|c|c|c|c|}
\hline Symbol & Variables & Unit & $(\mathbf{- 1})$ & $(\mathbf{0})$ & $(+\mathbf{+ 1})$ \\
\hline & & & & & \\
\hline$n$ & Spindle speed & $\mathrm{rpm}$ & 12000 & 15000 & 18000 \\
\hline$f$ & Feed rate & $\mathrm{m} / \mathrm{min}$ & 2 & 5 & 8 \\
\hline$d$ & Depth of cut & $\mathrm{mm}$ & 2 & 4 & 6 \\
\hline$r$ & Tool tip radius & $\mathrm{mm}$ & 8 & 10 & 12 \\
\hline
\end{tabular}


In this study, four parameters namely spindle speed, feed rate, depth of cut and tool radius were selected as input parameters while $R_{a}$ and $R_{z}$ were selected as output variables. (Table 2) shows the experimental parameters and their results.

Table 2: $L_{27}$ experimental design results.

\begin{tabular}{|c|c|c|c|c|c|c|}
\hline $\begin{array}{c}\text { Run } \\
\text { Order }\end{array}$ & $\begin{array}{c}\text { Spindle } \\
\text { Speed }\end{array}$ & Feed Rate & Depth of Cut & Tool Radius & Roughness $(\mu \mathrm{m})$ & \\
\hline & $(n, \mathbf{r p m})$ & $(f, \mathrm{~m} / \mathrm{min})$ & $(d, \mathbf{m m})$ & $(r, \mathbf{m m})$ & $\boldsymbol{R}_{a}$ & $\boldsymbol{R}_{z}$ \\
\hline 1 & 12000 & 2 & 2 & 8 & 3,06 & 14,24 \\
\hline 2 & 12000 & 2 & 4 & 10 & 4,29 & 17,16 \\
\hline 3 & 12000 & 2 & 6 & 12 & 5,33 & 21,32 \\
\hline 4 & 12000 & 5 & 2 & 10 & 7,25 & 29,30 \\
\hline 5 & 12000 & 5 & 4 & 12 & 4,89 & 21,56 \\
\hline 6 & 12000 & 5 & 6 & 8 & 6,37 & 27,48 \\
\hline 7 & 12000 & 8 & 2 & 12 & 8,74 & 36,96 \\
\hline 8 & 12000 & 8 & 4 & 8 & 9,18 & 35,72 \\
\hline 9 & 12000 & 8 & 6 & 10 & 8,53 & 33,12 \\
\hline 10 & 15000 & 2 & 2 & 10 & 4,59 & 18,36 \\
\hline 11 & 15000 & 2 & 6 & 12 & 8,89 & 36,56 \\
\hline 12 & 15000 & 2 & 6 & 8 & 6,59 & 23,36 \\
\hline 13 & 15000 & 5 & 2 & 12 & 6,37 & 26,32 \\
\hline 14 & 15000 & 5 & 4 & 8 & 5,03 & 21,18 \\
\hline 15 & 15000 & 5 & 6 & 10 & 6,98 & 28,72 \\
\hline 16 & 15000 & 8 & 2 & 8 & 7,11 & 29,32 \\
\hline 17 & 15000 & 8 & 4 & 10 & 6,96 & 28,62 \\
\hline 18 & 15000 & 8 & 6 & 12 & 6,81 & 28,12 \\
\hline 19 & 18000 & 2 & 2 & 12 & 6,07 & 25,18 \\
\hline 20 & 18000 & 2 & 4 & 8 & 3,96 & 16,42 \\
\hline 21 & 18000 & 2 & 6 & 10 & 8,59 & 35,36 \\
\hline 22 & 18000 & 5 & 2 & 8 & 3,72 & 12,16 \\
\hline 23 & 18000 & 5 & 4 & 10 & 4,29 & 18,14 \\
\hline 24 & 18000 & 5 & 6 & 12 & 8,29 & 34,12 \\
\hline 25 & 18000 & 8 & 2 & 10 & 3,40 & 14,54 \\
\hline 26 & 18000 & 8 & 4 & 12 & 4,89 & 20,34 \\
\hline 27 & 18000 & 8 & 6 & 8 & 4,01 & 17,14 \\
\hline
\end{tabular}

\section{RESULTS AND DISCUSSION}

In this section, two mathematical models used for optimization of the surface roughness were explained. The Taguchi orthogonal array integrated with response surface methodology and simulated angling algorithm for solving these models were detailed. In addition to these, the suggested models were tested by using verification test. Interactions between the variables were analyzed by using 3D surface plot. Finally, the results were 
compared and the suggested models were discussed.

\section{Developing the mathematical models}

The results were analyzed by conducting Minitab 17 software. Correlation of the experimental roughness value and the estimated values from the regression equation were shown in (Table 3 ). The experimental data were analyzed by the polynomial models such as linear, 2-way interaction and full quadratic models. The adequacy of models performed indicated that linear, interaction and quadratic models had lower $p$-value $(<0,05)$. (Table 3) displays the $R^{2}$ and Adjusted- $R^{2}$ values were found low in linear and interaction models. $R$-square and Adj- $R$-square values were described with Multiple Linear Regression Analysis (MLR). Quadratic models were calculated to have maximum $R^{2}$ and Adj- $R^{2}$ values. Hence the quadratic models were chosen for $R_{a}$ and $R_{z}$ in this study.

Table 3: Regression models, $R^{2}$ and $\mathrm{Adj}-R^{2}$.

\begin{tabular}{|c|c|c|c|}
\hline Regression model & $\boldsymbol{R}^{\mathbf{2}}$ & $\operatorname{Adj}-R^{2}$ & \\
\hline \multicolumn{4}{|l|}{ Linear } \\
\hline $\operatorname{Ra}(\mu \mathrm{m})=3,73-0,000193 n+0,167 f+0,367 d+0,292 r$ & 29,83 & 17,07 & \\
\hline $\mathrm{Rz}(\mu \mathrm{m})=13,5-0,000805 n+0,717 f+1,408 d+1,407 r$ & 10,04 & 4,32 & \\
\hline \multicolumn{4}{|l|}{ Linear + square } \\
\hline $\begin{array}{l}\mathrm{Ra}(\mu \mathrm{m})=-10,3+0,00180 n-0,011 f-1,26 d+0,81 r- \\
0,000000 n^{2}+0,0192 f^{2}+0,201 d^{2}-0,027 r^{2}\end{array}$ & 37,17 & 9,24 & \\
\hline $\begin{array}{l}\mathrm{Rz}(\mu \mathrm{m})=-68,3+0,00869 n+1,13 f-6,94 d+6,8 r- \\
0,000000 n^{2}-0,026 f^{2}+0,960 d^{2}-0,302 r^{2}\end{array}$ & 30,64 & 0,00 & \\
\hline \multicolumn{4}{|l|}{ Linear + interaction } \\
\hline $\begin{array}{l}\operatorname{Ra}(\mu \mathrm{m})=10,81-0,001040 n+4,553 f-1.127 d-1.425 r- \\
0,000191 n f+0,000115 n d+0.000134 n r-0,1357 f d- \\
0,0968 f r+0,0413 d r\end{array}$ & 90,50 & 84,55 & \\
\hline $\begin{array}{l}\operatorname{Rz}(\mu \mathrm{m})=78,5-0,00630 n+13,87 f-4,52 d-7,48 r- \\
0,000649 n f+0,000607 n d+0,000667 n r-0,382 f d- \\
0,179 f r-0,194 d r\end{array}$ & 82,58 & 71,69 & \\
\hline \multicolumn{4}{|l|}{ Full quadratic } \\
\hline $\begin{array}{l}\operatorname{Ra}(\mu \mathrm{m})=-2,69+0,000699 n+4,294 f-2,443 d-0,69 r- \\
0,000000 n^{2}+0,0146 f^{2}+0,1740 d^{2}-0,0376 r^{2}-0,000190 n f \\
+0,000112 n d+0,000133 n r-0,1306 f d- \\
0,0876 f r+0,0337 d r\end{array}$ & 95,91 & 91,15 & suggested \\
\hline $\begin{array}{l}\mathrm{Rz}(\mu \mathrm{m})=5,7+0,00128 n+17,32 f-10,61 d-3,58 r- \\
0,000000 n^{2}+0,0337 f^{2}+0,637 d^{2}-0,105 r^{2}-0,000733 n f \\
+0,000558 n d+0,000565 n r-0,5088 f d- \\
0,3810 f r+0,091 d r\end{array}$ & 96,12 & 95,93 & suggested \\
\hline
\end{tabular}

\section{Analysis of variance (ANOVA)}

Table 4 and Table 5 indicated variance analysis for $R_{a}$ and $R_{z}$. In this case $f, n, d, r, n^{2}, d^{2}, f d, f n, n d, n r, f r$ are significant factors. Contribution (PC \%) of each factor on the total variation is computed as percentage. The most effective parameters for $R_{a}$ and $R_{z}$ were interaction between feed rate and spindle speed, their contribution to the model values were $37,16 \%$ and $34,47 \%$, respectively, on the surface roughness. The coefficient of determination $R^{2}$ for the $R_{a}$ and $R_{z}$ of were $95,91 \%$ and $96,12 \%$ respectively. The Adjusted $R^{2}$ values for $R_{a}$ and $R_{z}$ were computed as $91,15 \%$ and $95,93 \%$ respectively. 
Table 4: ANOVA results for $R_{a}$.

\begin{tabular}{|l|l|l|l|l|l|}
\hline Source & DF & PC $(\%)$ & \multicolumn{1}{c}{ SS } & $\boldsymbol{F}$ & $\boldsymbol{P}$ \\
\hline Model & 14 & 95,91 & 86,82 & 20,12 & $0,000^{\mathbf{a}}$ \\
\hline Linear & 4 & 29,83 & 24,21 & 19,64 & $0,000^{\mathrm{a}}$ \\
\hline$n$ & 1 & 6,66 & 6,03 & 19,57 & $0,001^{\mathrm{a}}$ \\
\hline$f$ & 1 & 4,19 & 6,01 & 19,50 & $0,001^{\mathrm{a}}$ \\
\hline$d$ & 1 & 12,21 & 7,60 & 24,67 & $0,000^{\mathrm{a}}$ \\
\hline$r$ & 1 & 6,77 & 4,40 & 14,28 & $0,003^{\mathrm{a}}$ \\
\hline Square & 4 & 7,33 & 4,90 & 3,98 & $0,028^{\mathrm{a}}$ \\
\hline$n^{2}$ & 1 & 3,12 & 1,55 & 5,04 & $0,044^{\mathrm{a}}$ \\
\hline$f^{2}$ & 1 & 0,31 & 0,10 & 0,33 & 0,574 \\
\hline$d^{2}$ & 1 & 3,82 & 2,57 & 8,36 & $0,014^{\mathrm{a}}$ \\
\hline$r^{2}$ & 1 & 0,08 & 0,13 & 0,44 & 0,521 \\
\hline $2-$ Way Interaction & 6 & 58,75 & 53,18 & 28,76 & $0,000^{\mathrm{a}}$ \\
\hline$f^{*} d$ & 1 & 37,16 & 31,92 & 106,81 & $0,000^{\mathrm{a}}$ \\
\hline$n^{*} d$ & 1 & 3,91 & 5,11 & 16,59 & $0,002^{\mathrm{a}}$ \\
\hline$f^{*} n$ & 1 & 4,79 & 7,16 & 23,25 & $0,000^{\mathrm{a}}$ \\
\hline$n^{*} r$ & 1 & 9,17 & 7,27 & 23,60 & $0,000^{\mathrm{a}}$ \\
\hline$f^{*} r$ & 1 & 3,48 & 3,00 & 9,76 & $0,009^{\mathrm{a}}$ \\
\hline$d^{*} r$ & 1 & 0,24 & 0,21 & 0,70 & 0,420 \\
\hline Error & 12 & 4,09 & 3,69 & & \\
\hline Total & 26 & 100 & & & \\
\hline
\end{tabular}

The terms of $\left({ }^{a}\right)$ indicates significant parameter.

Table 5: ANOVA results for $R_{z}$

\begin{tabular}{|l|l|l|l|l|c}
\hline Source & $\mathbf{D F}$ & $\mathbf{P C}(\mathbf{\%})$ & \multicolumn{1}{|c}{ SS } & $\boldsymbol{F}$ & $\boldsymbol{P}$ \\
\hline Model & 14 & $98,12 \%$ & 1443,97 & 44,83 & $0,000^{\mathbf{a}}$ \\
\hline Linear & 4 & 32,95 & 439,20 & 47,72 & $0,000^{\mathbf{a}}$ \\
\hline$n$ & 1 & 7,13 & 104,93 & 45,61 & $0,000^{\mathbf{a}}$ \\
\hline$f$ & 1 & 4,87 & 106,88 & 46,45 & $0,000^{\mathbf{a}}$ \\
\hline$d$ & 1 & 11,30 & 111,66 & 48,53 & $0,000^{\mathbf{a}}$ \\
\hline$r$ & 1 & 9,65 & 110,32 & 47,95 & $0,000^{\mathbf{a}}$ \\
\hline Square & 4 & 6,08 & 64,06 & 6,96 & $0,004^{\mathbf{a}}$ \\
\hline$n^{2}$ & 1 & 2,58 & 20,84 & 9,06 & $0,011^{\mathbf{a}}$ \\
\hline$f^{2}$ & 1 & 0,16 & 0,55 & 0,24 & 0,634 \\
\hline$d^{2}$ & 1 & 3,31 & 34,49 & 14,99 & $0,002^{\mathbf{a}}$ \\
\hline$r^{2}$ & 1 & 0,03 & 1,05 & 0,46 & 0,512 \\
\hline $2-W a y$ Interaction & 6 & 59,09 & 869,54 & 62,99 & $0,000^{\mathbf{a}}$ \\
\hline$f^{*} d$ & 1 & 34,47 & 490,54 & 213,21 & $0,000^{\mathbf{a}}$ \\
\hline$n * d$ & 1 & 6,29 & 125,84 & 54,69 & $0,000^{\mathbf{a}}$ \\
\hline$f^{*} n$ & 1 & 5,63 & 129,68 & 56,36 & $0,000^{\mathbf{a}}$ \\
\hline$n^{*} r$ & 1 & 8,61 & 110,38 & 47,98 & $0,000^{\mathbf{a}}$ \\
\hline$f^{*} r$ & 1 & 3,99 & 56,80 & 24,69 & $0,000^{\mathbf{a}}$ \\
\hline$d^{*} r$ & 1 & 0,11 & 1,58 & 0,69 & 0,424 \\
\hline
\end{tabular}

The terms of $\left(^{a}\right)$ indicates significant parameter. 


\section{Evaluation of the models}

Normal Probability Plot (NPP) was applied to evaluate the data for normality. Residual is the mean difference between the observed value and the predicted or fitted value. If the residuals fall approximately along straight line, they are then normally distributed. In contrast, if the residuals do not fall fairly close to a straight line, they are then not normally distributed (Antony 2014). If the model is correct and if the assumptions are satisfied, the residuals should be structures; in particular, they should be unrelated to any other variable including the predicted response. Figure $3 \mathrm{a}$, Figure $3 \mathrm{~b}$, Figure $4 \mathrm{a}$ and Figure $4 \mathrm{~b}$ show that the residuals generally fall on a straight line implying that the errors were disturbed normally, meaning was the experimental data come from a normal population. As a result of the residuals, no unusual structures were apparent.
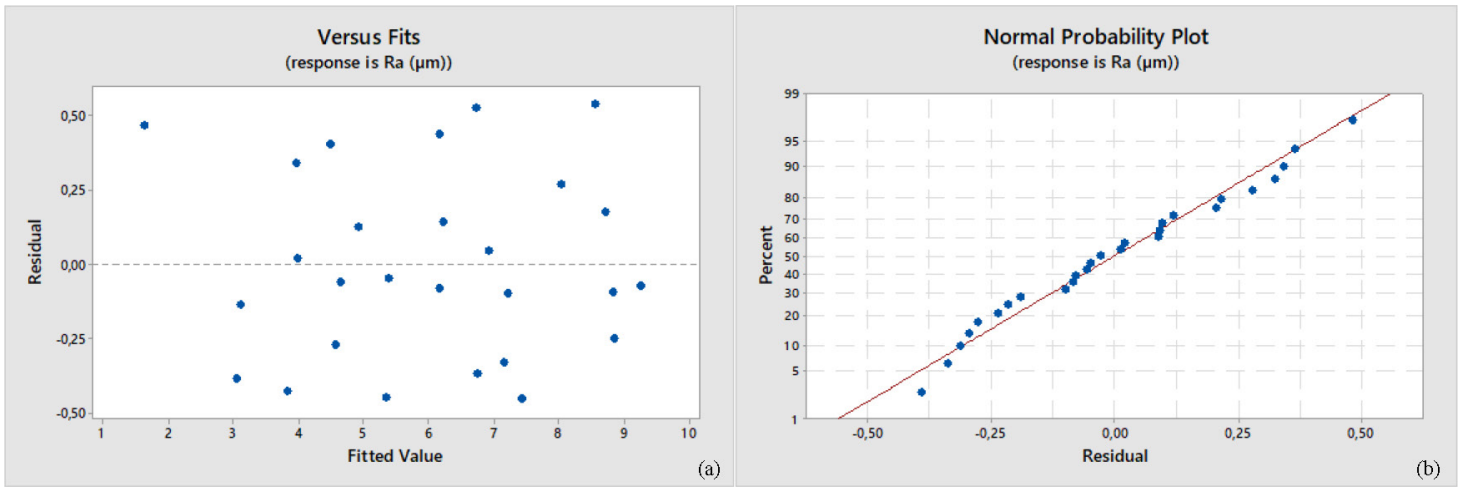

Figure 3: (a) Fitted value for $R_{a}$ and (b) NPP of residuals for $R_{a}$.
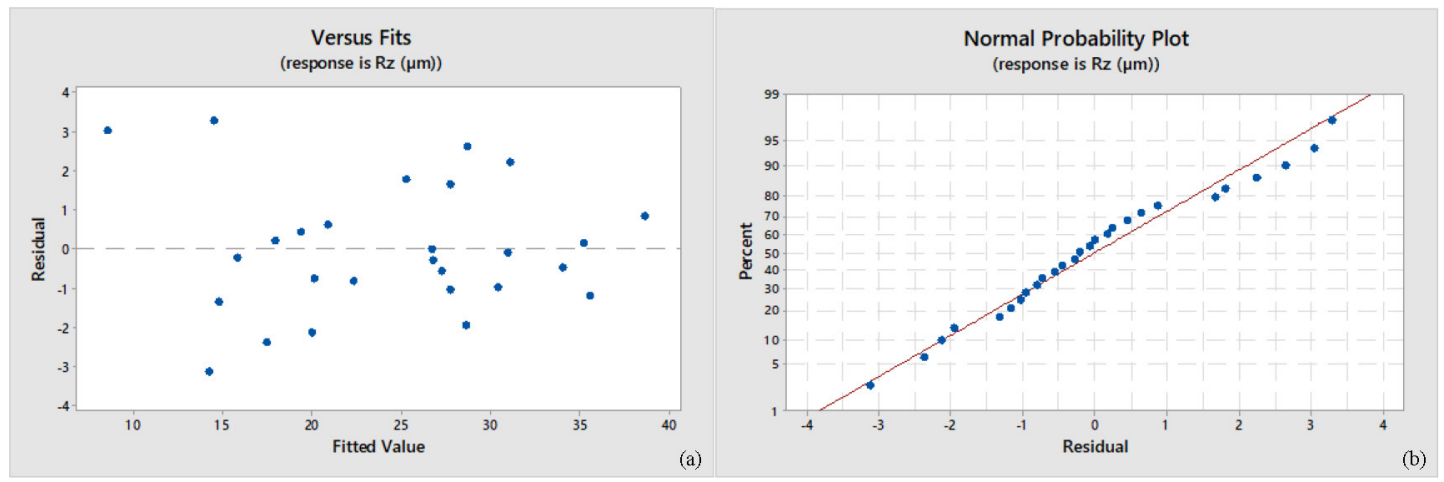

Figure 4: (a) Fitted value for $R_{\mathrm{z}}$ and (b) NPP of residuals for $R_{\mathrm{z}}$.

\section{Surface graphs and analysis}

Response surface provides the relationship between the factors with each other and the response variable. (Figure 5a) shows the surface plot for $R_{a}$ in CNC machining of wood material with three levels of feed rate and three levels of spindle speed for mid-level hold value of depth of cut with $4 \mathrm{~mm}$ and tool radius of $10 \mathrm{~mm}$. The surface roughness increased with decreases the spindle speed and increases the feed rate. (Figure $5 \mathrm{~b}$ ) shows the surface plot for $R_{a}$ in CNC machining of wood material with three levels of depth of cut and three levels of tool radius for mid-level hold value with feed rate of $5 \mathrm{~m} / \mathrm{min}$ and spindle speed of $15000 \mathrm{rpm}$. The surface roughness decreased with a decrease of tool radius and depth of cut. (Figure $5 \mathrm{c}$ ) shows the surface plot for $R_{a}$ in CNC machining of wood material with three levels of feed rate and three levels of tool radius for mid-level hold value with spindle speed of $15000 \mathrm{rpm}$ and $4 \mathrm{~mm}$ depth of cut. The surface roughness increased with the increase in tool radius and feed rate. (Figure $5 \mathrm{~d}$ ) shows the surface plot for $R_{a}$ in CNC machining of wood material with three levels of feed rate and three levels of depth of cut for mid-level hold value with spindle speed of $15000 \mathrm{rpm}$ and tool radius of $10 \mathrm{~mm}$. The surface roughness increased with the increase in feed rate and depth of cut. (Figure 5e) shows the surface plot for $R$ in CNC machining of wood material with three levels of spindle speed and three levels of tool radius for mid-level hold value with $5 \mathrm{~m} / \mathrm{min}$ of feed rate and depth of 
cut of $4 \mathrm{~mm}$. The surface roughness increased with the increase in tool radius and decreases with spindle speed. (Figure 5f) shows the surface plot for $R$ in CNC machining of wood material with three levels of depth of cut and three levels of spindle speed for mid-level hold value with $5 \mathrm{~m} / \mathrm{min}$ of feed rate and tool radius of $10 \mathrm{~mm}$. The surface roughness increased with the increase in depth of cut and decrease with spindle speed.

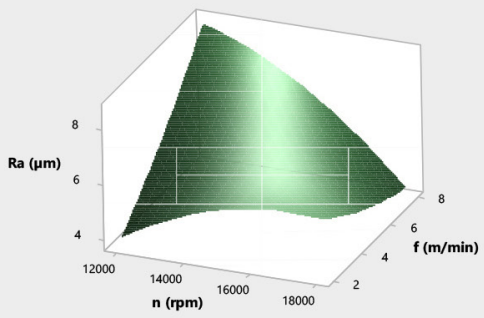

(a)

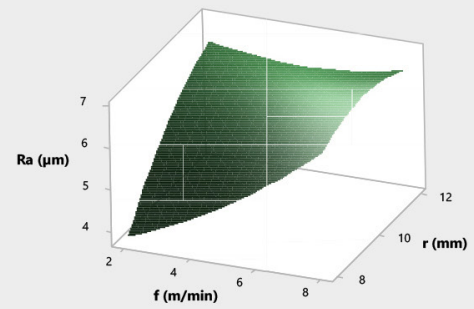

(c)

(e)
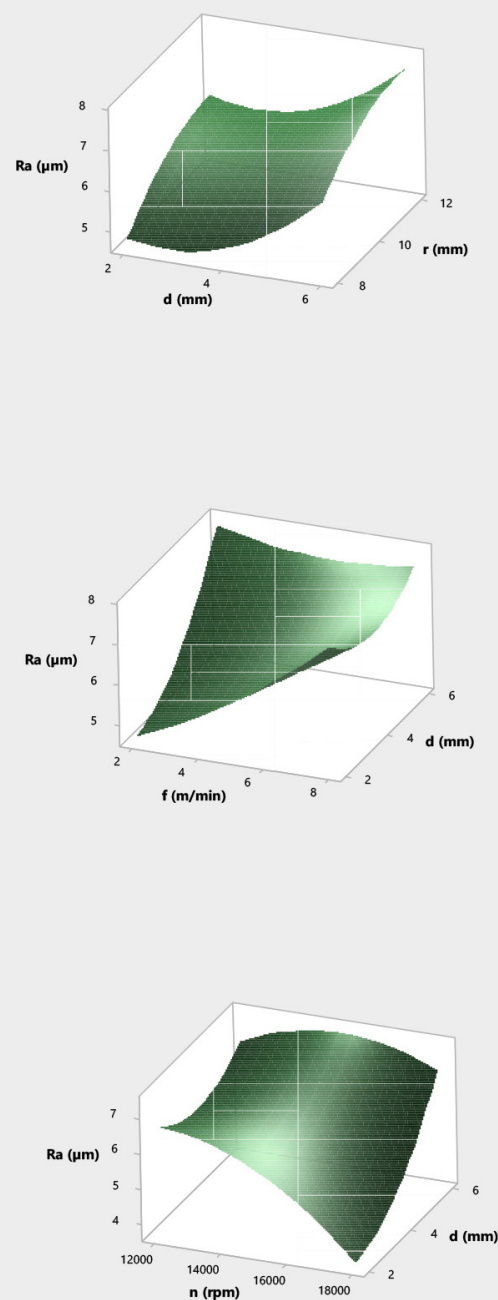

(b)

(d)

(f)

Figure 5: According to 3D surface plots (a, b, c, d, e, f) of different values processing parameters for $R_{a}$.

(Figure 6a) shows the surface plot for $R_{\mathrm{z}}$ in CNC machining of wood material with three levels of feed rate and three levels of spindle speed for mid-level hold value of depth of cut with $4 \mathrm{~mm}$ and tool radius of 10 $\mathrm{mm}$. The surface roughness increased with decreases the spindle speed and increases the feed rate. (Figure $6 \mathrm{~b}$ ) shows the surface plot for $R_{a}$ in CNC machining of wood material with three levels of depth of cut and three levels of tool radius mid-level hold value with feed rate of $5 \mathrm{~m} / \mathrm{min}$ and spindle speed of $15000 \mathrm{rpm}$. The surface roughness decreased with a decrease of tool radius and depth of cut. (Figure 6c) shows the surface plot for $R_{a}$ CNC machining of wood material with three levels of feed rate and three levels of tool radius for mid-level hold value with $15000 \mathrm{rpm}$ of spindle speed and depth of cut of $4 \mathrm{~mm}$. The surface roughness increased with the increase in tool radius and feed rate. (Figure 6d) shows the surface plot for $R_{a}$ in CNC machining of wood material with three levels of feed rate and three levels of depth of cut for mid-level hold value with $15000 \mathrm{rpm}$ of spindle speed and tool radius of $10 \mathrm{~mm}$. The surface roughness increased with the increase in feed rate and 
depth of cut. (Figure 6e) shows the surface plot for $R$ in CNC machining of wood material with three levels of spindle speed and three levels of tool radius for mid-level hold value with $5 \mathrm{~m} / \mathrm{min}$ of feed rate and depth of cut of $4 \mathrm{~mm}$. The surface roughness increased with the increase in tool radius and decreases with spindle speed. (Figure 6f) shows the surface plot for $R_{a}$ in CNC machining of wood material with three levels of depth of cut and three levels of spindle speed for mid-level hold value with $5 \mathrm{~m} / \mathrm{min}$ of feed rate and tool radius of $10 \mathrm{~mm}$. The surface roughness increased with the increase in depth of cut and decrease with spindle speed.

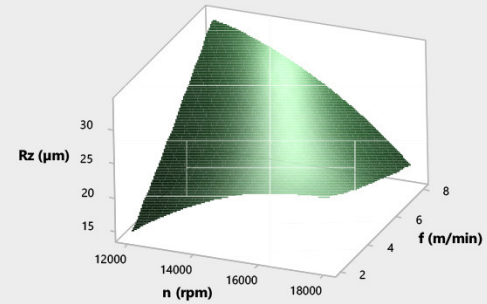

(a)
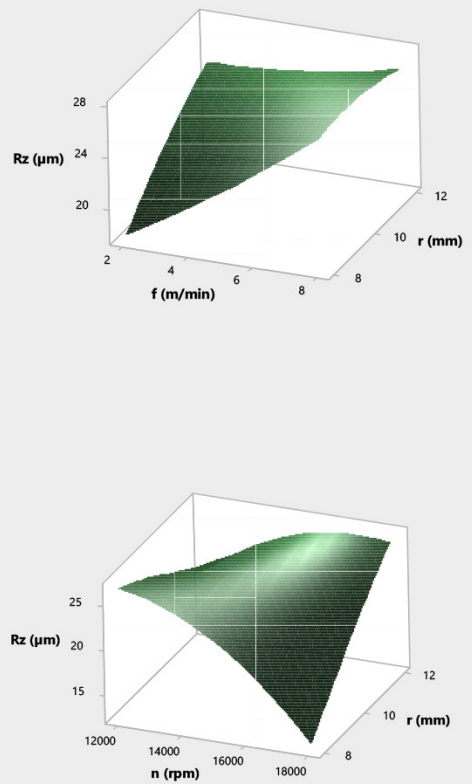

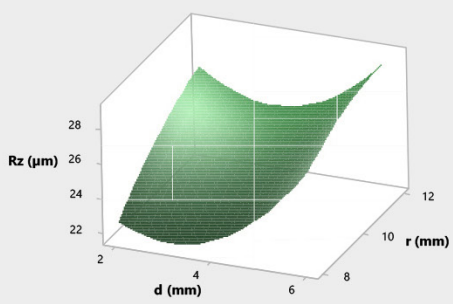

(b)

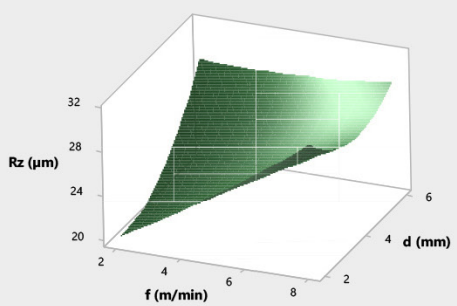

(d)

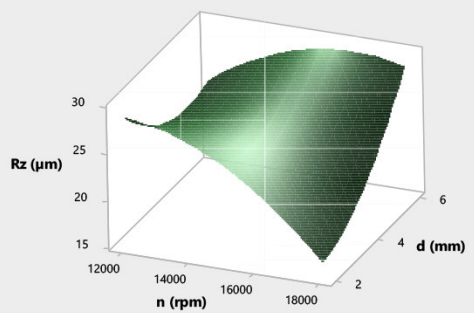

(f)

Figure 6: According to 3D surface plots (a, b, c, d, e, f) of different values processing parameters for $R_{\mathrm{z}}$. 


\section{Parameter optimization}

The aim of present work was to find the optimal processing levels leading to minimum values of $R_{a}$ and $R_{z}$. Two second order mathematical models were created by using RSM. Moreover, backward elimination method was carried out for determining the non-significant terms in mathematical models. To formulate the optimization problem, proposed models were presented in Equation 4 and Equation 5:

$$
\begin{aligned}
& \text { Minimize } R a=9,42-0,001069 n+4,510 f-0,710 d-1,251 r-0,000186 n f \\
& +0,000115 n d+0,000134 n r-0,1374 f d-0,0990 f r \\
& \text { Minimize } R z=11,2+0,00134 n+17,56 f-9,68 d-5,29 r-0,000000 n^{2} \\
& +0,635 d^{2}-0,000722 n f+0,000559 n d+0,000566 n r-0,5116 f d-0,3860 f r
\end{aligned}
$$

These models were solved with two different methods namely, desirability function and simulated angling algorithm. Firstly, the desirability function was applied to determine the initial optimum parameter levels. Then, the results were used the initial point for the simulated angling algorithm. The functions terms were optimized within the specified range. The range of machining conditions was selected and given as follows Equation 6a, Equation 6b, Equation 6c, Equation 6d:

Within ranges of processing parameters,

$$
\begin{array}{rl}
2 & \leq f \leq 8 \\
12000 \leq n \leq 18000 & 6(\text { a) } \\
2 & 6 \text { (b) } \\
8 & \leq r \leq 12 \quad 6(\mathrm{c})
\end{array}
$$

The limitations of cutting variables value of spindle speed, feed rate, depth of cut and tool radius were $12000 \mathrm{rpm}, 2 \mathrm{~m} / \mathrm{min}, 2 \mathrm{~mm}$ and $8 \mathrm{~mm}$ as lower limits while $18000 \mathrm{rpm}, 8 \mathrm{~m} / \mathrm{min}, 6 \mathrm{~mm}$ and $12 \mathrm{~mm}$ were selected as upper limits, respectively.

\section{Parameter optimization by using desirability function (DF)}

This is commonly applied engineering problems for the optimization process. The function value is range 0 from 1 . In the present work, selected quality characteristic was smaller-the-better for minimizing the surface roughness value. This equation was presented in Equation 7: 


$$
d_{i}= \begin{cases}1 & y_{i}<T \\ \left(\frac{U-y_{i}}{U-T}\right)^{\omega} & T \leq y_{i} \leq U \\ 0 & y_{i}>U\end{cases}
$$

Where $T$ symbolizes the target value of the $i$ th response, $y_{i}, L$ symbolizes the acceptable lower limit value, $U$ symbolizes the acceptable upper limit, for this response and $W$ represents the weight. In (Table 6), optimal machining parameters for minimizing $R_{a}$ was found with spindle speed of $17450 \mathrm{rpm}$, feed rate of $2,9 \mathrm{~m} / \mathrm{min}$, tool radius of $8,0 \mathrm{~mm}$ and depth of cut of $2,0 \mathrm{~mm}$. optimal machining parameters for minimizing $R_{z}$ was spindle speed of $16990 \mathrm{rpm}$, feed rate of $2,0 \mathrm{~m} / \mathrm{min}$, tool radius of $8,0 \mathrm{~mm}$ and depth of cut of $2,0 \mathrm{~mm}$.

Table 6: Taguchi- DF results for $R_{a}$ and $R_{z}$.

\begin{tabular}{|c|c|c|c|c|c|c|c|}
\hline Response & Target & \multicolumn{3}{|c|}{ Optimal conditions } & & \multirow{2}{*}{$\begin{array}{c}\text { Predicted } \\
(\boldsymbol{\mu m})\end{array}$} & Desirability \\
\hline & & $\boldsymbol{f}(\mathbf{m m} / \mathbf{m i n})$ & $\boldsymbol{n}(\mathbf{r p m})$ & $\boldsymbol{d}(\mathbf{m m})$ & $\boldsymbol{r ( m m )}$ & & \\
\hline $\mathrm{Ra}(\mu \mathrm{m})$ & Min. & 2,9 & 17450 & 2,0 & 8,0 & 3,1287 & 0,988 \\
\hline $\mathrm{Rz}(\mu \mathrm{m})$ & Min, & 2,0 & 16990 & 2,0 & 8,0 & 11,254 & 1,000 \\
\hline
\end{tabular}

From the Figure 7 and Figure 8, the optimum values for $R_{a}$ and $R_{z}$ were 3,1287 $\mu \mathrm{m}$ and $11,254 \mu \mathrm{m}$, respectively.

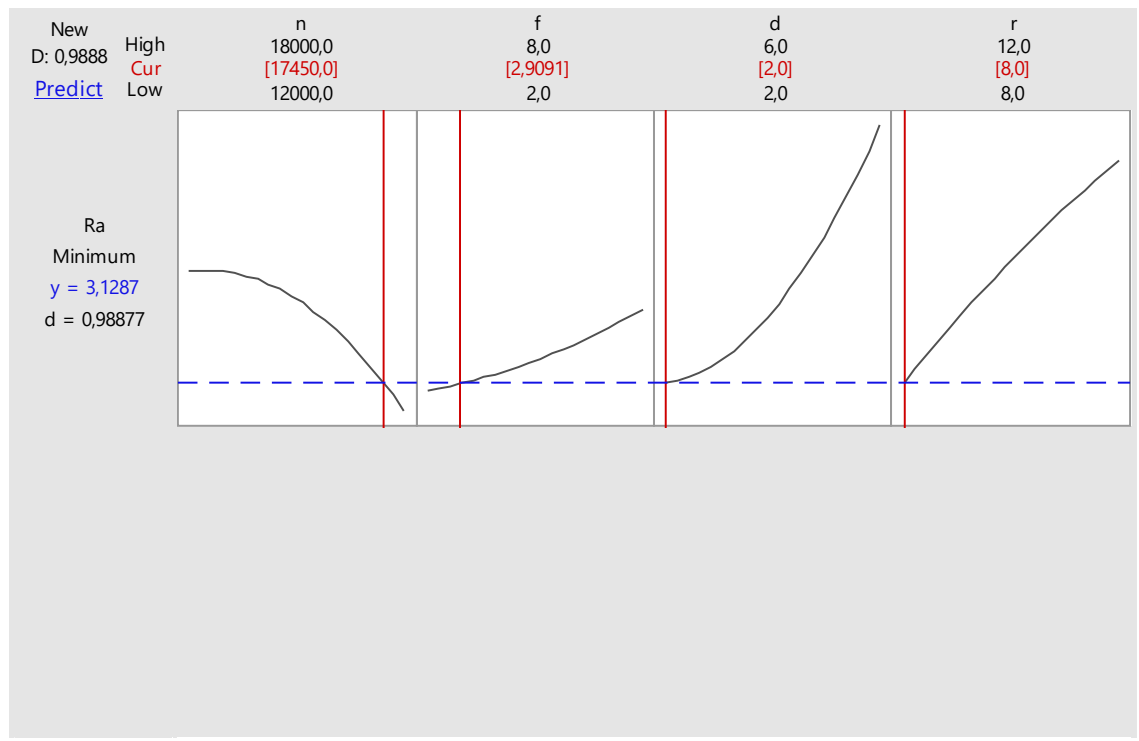

Figure 7: Response optimization plot for $R_{a}$. 


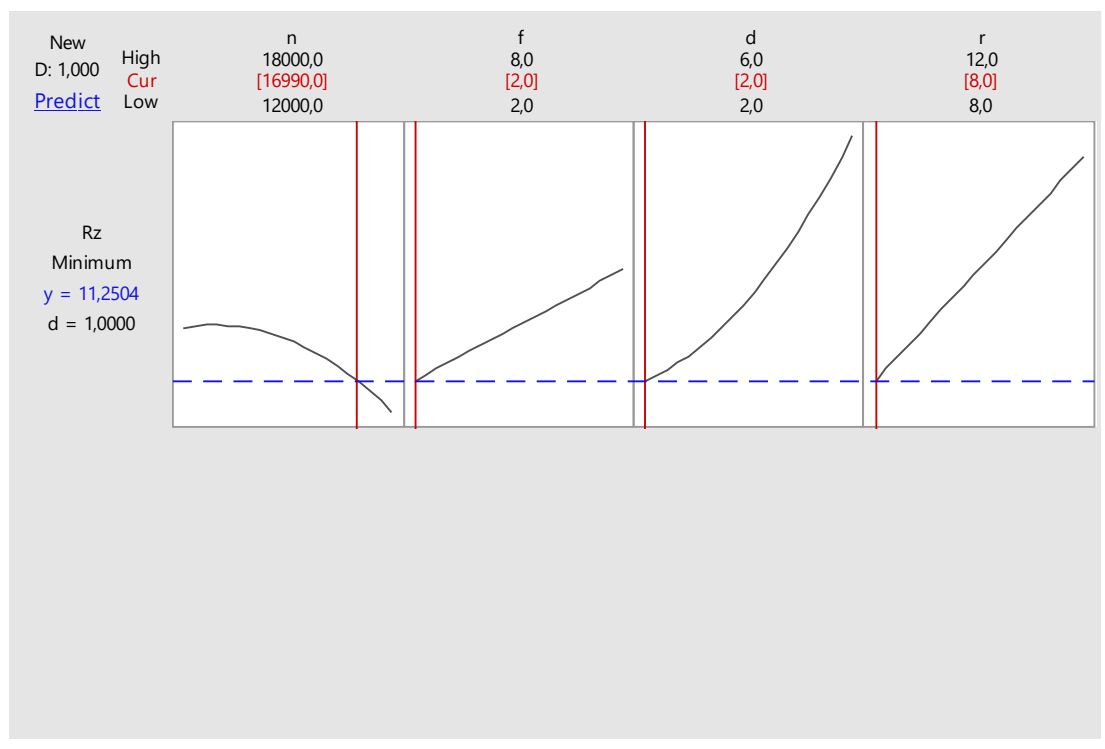

Figure 8: Response optimization plot for $R_{z}$.

\section{Parameter optimization by simulated angling algorithm (SA)}

In this study, simulated angling algorithm was applied to optimize the machining parameters. It is the one of the most useful metaheuristics method for solving the engineering problems due to easy application. It is a widely used method in solving optimization problems. SA is a probabilistic and single solving method based on the annealing process of metallurgy. The algorithm starts with an initial point using random solution, $S$, then a simple random change is produced to actual solution $\left(S^{\prime}\right)$. Obtained solution was compared with new solution by creating the objective function. The new solution is accepted when the objective $f\left(S^{\prime}\right)$ is smaller than other objective. This solution is recognized with a probability of $\exp ^{-\left(f\left(S^{\prime}\right) f(S)\right) / T}$. The term of $T$ is a control variable and it is reduced from a relatively to near zero. SA was applied by solving the fitness function formulated in Equation 4 and Equation 5. In (Table 1), the range of processing variables was defined to solve the surface roughness problem. Initial points of SA are given in (Table 7).

Table 7: Initial points of SA for $R_{a}$ and $R_{z}$.

\begin{tabular}{|c|c|c|}
\hline Variables & $\boldsymbol{R}_{\boldsymbol{a}}$ & $\boldsymbol{R}_{\boldsymbol{z}}$ \\
\hline Spindle speed & 17450 & 16990 \\
\hline Feed rate & 2,90 & 2,0 \\
\hline Depth of cut & 2,0 & 2,0 \\
\hline Tool radius & 8,0 & 8,0 \\
\hline
\end{tabular}

Matlab optimization toolbox was used to solve the surface roughness problem. The values of the SA parameters such as annealing function, reannealing interval, temperature function, initial point of temperature, and probability function. Matlab software was used to determine the best optimal results using different simulated angling parameters. The best solution of these parameters was given in (Table 8). 
Table 8: Selection of SA parameters to determine the optimal solution.

\begin{tabular}{|c|c|c|}
\hline \multirow{2}{*}{ SA Parameters } & \multicolumn{2}{|c|}{ Selected function and values } \\
\cline { 2 - 3 } & $\boldsymbol{R}_{\boldsymbol{a}}$ & $\boldsymbol{R}_{\boldsymbol{z}}$ \\
\hline Annealing function & Boltzmann annealing & Fast annealing \\
\hline Reannealing interval & 100 & 100 \\
\hline Temperature function & Exponential & Exponential \\
\hline Initial temperature & 100 & 100 \\
\hline Probability function & SA & SA \\
\hline Data type & Double & Double \\
\hline
\end{tabular}

Minimum $R_{a}$ value was resulted with spindle speed of $17377 \mathrm{rpm}$, feed rate of 2,012 $\mathrm{m} / \mathrm{min}$, tool radius of $8,00 \mathrm{~mm}$ and depth of cut of 2,009 $\mathrm{mm}$ using simulated angling algorithm. Minimum $R_{z}$ value was obtained spindle speed of $16980 \mathrm{rpm}$, feed rate of 2,004 m/min, tool radius of $8,001 \mathrm{~mm}$ and depth of cut of $2,003 \mathrm{~mm}$ using simulated angling algorithm. The results of Matlab SA toolbox were given in Figure 9a, Figure 9b, Figure 10a, Figure 10b. According to Figure 9a, Figure 10a, the best fitness values for $R_{a}$ and $R_{z}$ were $2,474 \mu \mathrm{m}$ and 10,674 $\mu \mathrm{m}$, respectively. In the Figure 10a, Figure 10b, the optimal solutions of $R_{a}$ and $R_{z}$ were performed at the 3106 and 4712 iteration, respectively.
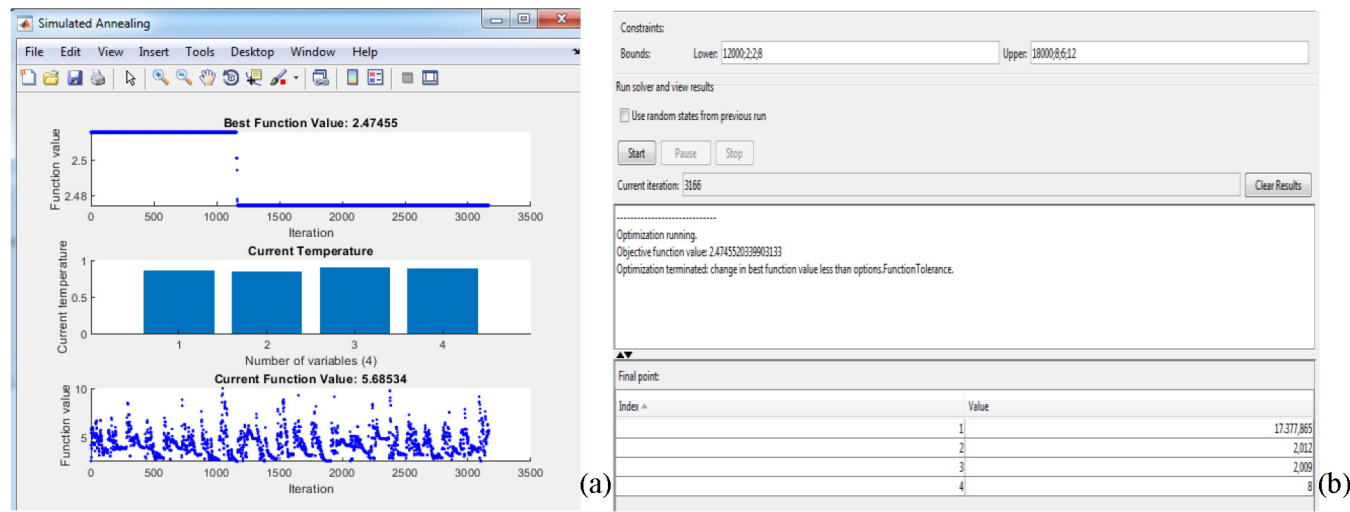

Figure 9: (a) Variation of fitness function and best individuals (b) Converged values of parameters.
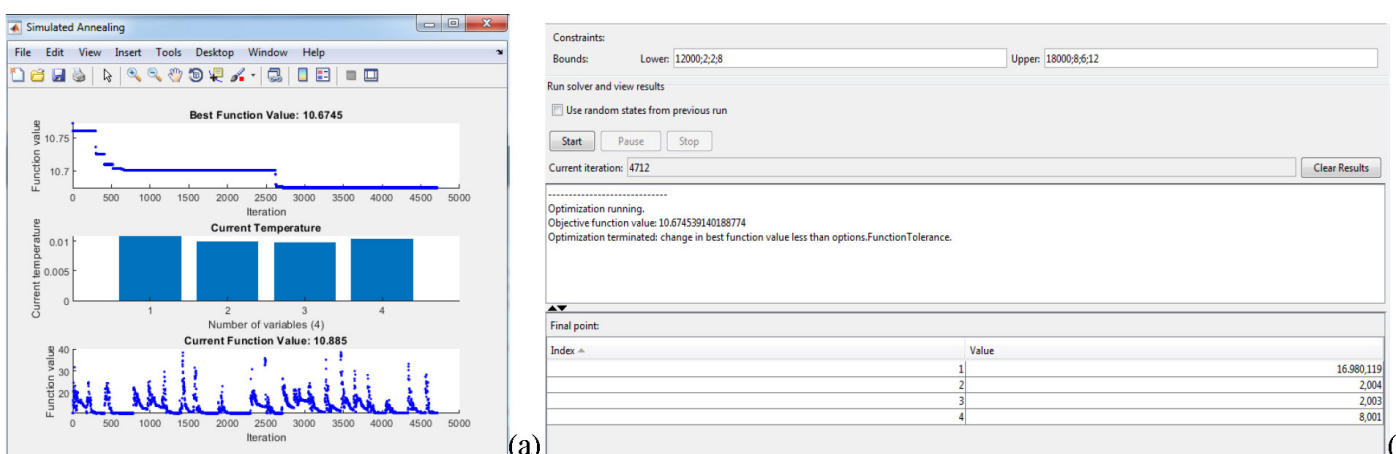

(a)

(b)

Figure 10: (a) Variation of fitness function and best individuals (b) Converged values of parameters. 


\section{Comparison of results}

The Taguchi orthogonal design based desirability function and desirability function integrated simulated angling algorithm results were given in (Table 9). From the (Table 9), the minimum $R_{a}$ and $\mathrm{R}_{\mathrm{z}}$ values were $2,474 \mu \mathrm{m}$ and $10,674 \mu \mathrm{m}$. For this reason, the RSM-DF-GA approach should present an efficient methodology in order to minimize the surface roughness.

Table 9: Results of experiments and optimum values by Taguchi-RSM-DF and Taguchi-RSM-DF-SA models for $R_{a}$ and $R_{z}$

\begin{tabular}{|c|c|c|c|c|c|c|}
\hline \multirow{2}{*}{ Proposed models } & \multicolumn{4}{|c|}{ Optimal conditions } & \multirow{2}{*}{$\begin{array}{c}\text { Response } \\
(\mu \mathrm{m}) \\
\boldsymbol{R}_{a}\end{array}$} & \multirow[b]{2}{*}{$\boldsymbol{R}_{z}$} \\
\hline & $f(\mathrm{~mm} / \mathrm{min})$ & $n$ (rpm) & $d(\mathrm{~mm})$ & $r(\mathbf{m m})$ & & \\
\hline Taguchi-RSM-DF & 2,900 & 17450 & 2,000 & 8,000 & 3,128 & - \\
\hline $\begin{array}{c}\text { Taguchi-RSM-DF- } \\
\text { SA }\end{array}$ & 2,012 & 17377 & 2,009 & 8,000 & 2,474 & - \\
\hline Taguchi-RSM-DF & 2,000 & 16990 & 2,000 & 8,000 & - & 11,254 \\
\hline $\begin{array}{c}\text { Taguchi-RSM-DF- } \\
\text { SA }\end{array}$ & 2,004 & 16980 & 2,003 & 8,001 & - & 10,674 \\
\hline
\end{tabular}

\section{CONCLUSIONS}

In this work, effect of machining of Cedar of Lebanon pine (Cedrus libani), the surface roughness characteristics were investigated. The optimization process was adopted by a combined approach of $L_{27}$ orthogonal array based simulated angling algorithm. A three-levels and four factors experimental design were applied to identify the most significant factors, desirability function and simulated angling algorithm were used to optimize the machining procedure. The results can be summarized as follows:

Different from other studies in the literature, two mathematical models were developed based on $L_{27}$ orthogonal design and RSM method that the method has not been used to determine optimum CNC parameters for wood material.

The $R_{a}$ and $R_{z}$ were influenced by interaction between feed rate and spindle speed with PCR (\%) of 37,16 $\%$ and $34,47 \%$, respectively.

Two different mathematical models were applied by using quadratic models. Moreover, backforward elimination method was applied to determine the non-significant variables. The non-significant terms were removed in these models.

Minimum $R_{a}$ value was obtained spindle speed of $17377 \mathrm{rpm}$, feed rate of 2,012 $\mathrm{m} / \mathrm{min}$, tool radius of 8 $\mathrm{mm}$ and depth of cut of 2,009 $\mathrm{mm}$ by using desirability function based simulated angling algorithm. For $R_{z}$

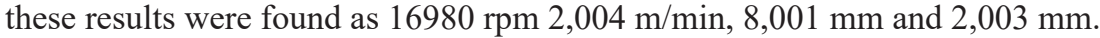


In this study, these models can be regarded as a method experimentally and statistically for the analysis, modeling optimization of $\mathrm{CNC}$ machining operations and can applied wood cutting process.

\section{ACKNOWLEDGMENTS}

The authors thank to TUBITAK (The Scientific and Technological Research Council of TURKEY) for support and contributions during the doctoral thesis.

\section{REFERENCES}

Antony, J. 2014. Design of experiments for engineers and scientists. 2th edn. Elsevier: London.

Asiltürk, I.; Neseli, S.; Ince, A.M. 2016. Optimization of parameters affecting surface roughness of Co28Cr6Mo medical material during CNC lathe machining by using the Taguchi and RSM methods. Measurement 78:120-128.

Azhiri, R.B; Teimouri, R.; Baboly, M.G.; Leseman, Z. 2014. Application of Taguchi, ANFIS and grey relation analysis for studying, modeling and optimization of wire EDM process while using gaseous media. Int $J$ Adv Manuf Technol 71(1-4):279-295.

Bao, X.; Ying, J.; Cheng, F.; Zhang, J.; Luo, B.; Li, L.; Liu, H. 2017. Research on neural network model of surface roughness in belt sanding process for Pinus koraiensis. Measurement 115:11-18.

Bharathi, S.R; Baskar, N. 2011. Particle swarm optimization technique for determining optimal machining parameters of different work piece materials in turning operation. The International Journal of Advanced Manufacturing Technology 54 (5-8):445-463.

Coelho, C.L.; Carvalho, L.M.H.; Martins, J.M.; Costa, V.A.V.; Masson, D.; Meausooner, P.J. 2008. Method for evaluating the influence of wood machining conditions on the objective characterization and subjective perception of a finished surface. Wood Sci Technol 42(3):181-195.

Davim, J.P.; Clemente, V.C.; Silva, S. 2009. Surface roughness aspects in milling MDF (medium density fiberboard). Int J Adv Manuf Technol 40(1-2):49-55.

Deepanraj, B.; Sivasubramanian, V.; Jayaraj, S. 2017. Multi-response optimization of process parameters in biogas production from food waste using Taguchi-Grey relational analysis. Energy Conversion and Management 141:429-438.

Gaitonde, V.N.; Karnik, S.R.; Davim, J.P. 2008. Taguchi multi-performance characteristics optimization in drilling of medium density fibreboard (MDF) to minimize delamination using utility concept. J Mater Process Tech 196(1-3):73-78.

Hazir, E.; Erdinler, E.S.; Koç, K.H. 2018. Optimization of CNC cutting parameters using design of experiment (DOE) and desirability function. Journal of Forestry Research 29(5):1423-1434.

Hazir, E.; Ozcan, T.2019. Response surface methodology integrated with desirability function and genetic algorithm approach for the optimization of CNC machining parameters. Arabian Journal for Science and Engineering 44(3):2795-2809.

Hiziroglu, S.; Kosonkorn, P. 2006. Evaluation of surface roughness of Thai medium density fiberboard (MDF). Building and Environment 41(4):527-533. 
International Organization for Standardization. ISO. 1997. Geometrical product specifications (GPS) surface texture: profile method-terms, definitions, and surface texture profile method terms, definitions and surface texture parameters. ISO 4287. 1997. International Organization for Standardization: Geneva.

Kant, G.; Sangwan, K.S. 2014. Prediction and optimization of machining parameters for minimizing power consumption and surface roughness in machining. Journal of Cleaner Production 83:151-164.

Koç, K.H.; Erdinler, E.S.; Hazir, E.; Öztürk, E. 2017. Effect of CNC application parameters on wooden surface quality. Measurement 107:12-18.

Magoss, E. 2008. General regularities of wood surface roughness. Acta Silv Lign Hung 4:81-93.

Mahes, G.; Muthu, S.; Devadasan, S.R. 2015. Prediction of surface roughness of end milling operation using genetic algorithm. Int J Adv Manuf Technol 77(1-4):369-381.

Majumder, H.; Paul, T.R.; Dey, V.; Dutta, P.; Saha, A. 2017. Use of PCA-grey analysis and RSM to model cutting time and surface finish of Inconel 800 during wire electro discharge cutting. Measurement 107:19-30.

Ozdemir, T.; Hiziroglu, S. 2007. Evaluation of some sanding factors on the surface roughness of particleboard. Silva Fennica 41(2):373-378.

Philbin, P.; Gordon, S. 2006. Recent research on the machining of wood-based composite materials. International Journal of Machining and Machinability of Materials 1(2):186-201.

Prakash, S.; Palanikumar, K. 2010. Modeling for prediction of surface roughness in drilling MDF panels using response surface methodology. J Compos Mater 45(16):1639-1646.

Rao, R.V.; Kalyankar, V.D. 2013. Parameter optimization of modern machining process using teaching-learning-based optimization algorithm. Engineering Application of Artificial Intelligence 26(1): 524-531.

Rao, V.K.; Murthy, N.S.G.B.P. 2018. Modeling and optimization of tool vibration and surface roughness in boring of steel using RSM, ANN and SVM. J Intell Manuf 29(7):1533-1543.

Ratnasingman, J.; Scholz, F. 2006. Optimal surface roughness for high-quality on Rubberwood. Holz-als Roh-und Werkstoff 64(4): 343-345.

Samanta, B. 2009. Surface roughness prediction in machining using soft computing. International Journal of Computer Integrated Manufacturing 22(3):257-266.

Sarikaya, M.; Güllü, A. 2016. Taguchi design and response surface methodology based analysis of machining parameters in CNC turning under MQL. Journal of Cleaner Production 65:604-616.

Selaimia, A.A.; Yallese, M.A.; Bensouilah, H.; Meddour, I.; Khattabi, R.; Mabrouki, T. 2017. Modeling and optimization in dry face milling of X2CrNi18-9 austenitic stainless steel using RSM and desirability approach. Measurement 107:53-67.

Sofuoglu, D.S. 2017. Determination of optimal machining parameters of massive wooden edge glued panels which is made of Scots pine (Pinus sylvestris L.) using Taguchi design method. Eur $J$ Wood Prod 75(1):33-42.

Taguchi, G.; Chowdhury, S.; Wu, Y. 2005. Taguchi's quality engineering handbook. Wiley: Hoboken.

Wilkowski, J.; Czarniak, P.; Grześkiewicz, M. 2011. Machinability evaluation of thermally modified wood using the Taguchi technique. In: COST Action FP0904 Workshop Mechano-chemical transformations of wood during Thermo-Hydro-Mechanical processing: 109-111.

Yang, W.H.; Tarng, Y.S. 1998. Design optimization of cutting parameters for turning operations based on the Taguchi Method. J Mater Process Technol 84(1-3):122-129.

Zhou, Y.; Gong, Y.; Zhu, Z.; Gao, Q.; Wen, X. 2016. Modeling and optimization of surface roughness from micro grinding of nickel-based single crystal super alloy using the response surface methodology and genetic algorithm. Int Adv Manuf Technol 85(9-12):2607-2622. 
Zhou, Y.; Ren J.;Yao, C. 2017. Multi-objective optimization of multi-axis ball-end milling Inconel 718 via grey relational analysis coupled with RBF neural network and PSO algorithm. Measurement 102:271-285. 